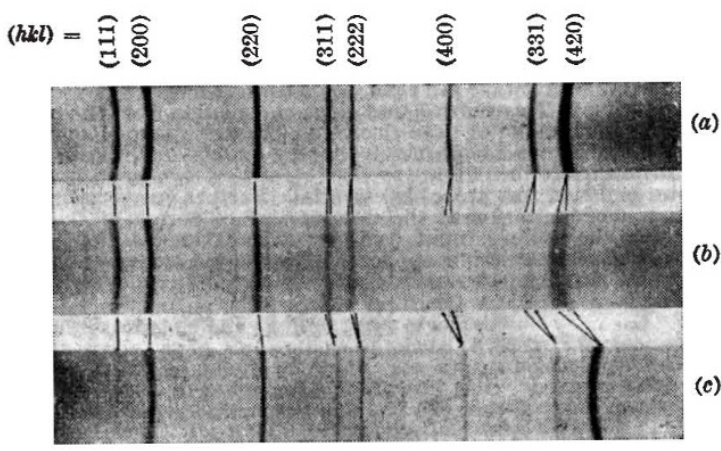

a . Ferrous oxide formed on pure iron.

$a$. Ferrous oxide formed on pure iron.
c. Magnesium oxide". " iron covered with magnesium oxide.

rosion values; this may not, however, be conclusive, because macro scopic factors, such as porosity and intergranular cracking of the oxide layers, may well mask the more subtle lattice effect. In these test the complete embodiment of magnesia in the wilstite structure was also manifest from the physical appearance of the scale (colour, layering adherence, etc.), which was indistinguishable, whether magnesium oxide had been present or not.

The purpose of the present note is chiefly to report the existence of 'impregnated' ferrous oxide ; its physical properties have not as yet been fully studied. It may not, however, be irrelevant to consider the phenomena from the point of vlew of catalysis, in that catic sction is known to depend in many cases on the adsorption of foreign atoms in the crystal lattice, and defect structures such as of are favourable.

The B.S.A. Group Research Centre,

H. J. GoLDsohmidr.

Nov. 30 .

${ }_{1}^{1}$ Wagner, C., Trans. Faraday Soc., 34, 851 (1938).

2 Pfeil, L. B., J. Iron and Steel Inst., 119, 501 (1929); 123, 237 (1931).

Goldschmidt, H. J., J. Iron and Steel Inst., ii, 146, $157 \mathrm{P}$ (1942).

\section{X-Ray Examination of Hail}

DURING a hailstorm on February 1,1946 , we collected a number of stones, each approximately $9 \mathrm{~mm}$. across, and having the familia concentric formation of hard hail. These were kept in a domestic refrigerator, and sections, 1-2 mm. thick, of three of them were photographed in a circular camera at about $-5^{\circ} \mathrm{C}$., using a $1 \mathrm{~mm}$. diameter cylindrical beam of copper $K \alpha$ radiation. The photographs were those of single or (in one case) several single crystals of tridymite ice-the normal form. Neighbouring portions of one hailstone showed no observable difference in orientation. Untortunately, an accidental interruption of the refrigeration allowed the stones to melt before there concentric layers.

Good single crystals of ice can be grown in cold weather within an hour or two, but an abrupt cooling of water, say, by a freezing mixture or by liquid air, usually results in the formation of a coarse powder. Our experiments show, therefore, that the hailstones were probably formed by a relatively slow fall of temperature in the air through which they passed.

\section{KATHLEEN LONSDALE.}

\section{Royal Institution,
Albemarle Street, W.1.}

\section{Supporting Electrolytes in Polarographic Analysis}

BUFFrR solutions containing ammonia and an ammonium salt have been widely used as supporting electrolytes in polarographic analysis. Tyler and Dean ${ }^{1}$ found that the total ionic concentration of such a solution might be increased without greatly affecting the reduction steps of copper and zinc, so that slight variations of concentration were less important than in other supporting electrolytes. Lingane ${ }^{2}$ reported that the diffusion current constants of copper, cadmium and zine in ammoniacal solutions were higher than those found in decinormal potassium chloride. From his data, it may be calculated that the mobilities of the complex ammino-cations in ammoniacal
solution are considerably greater than those of the aquo-ions, and correspond to an apparent ionic radius less than $3 \mathrm{~A}$

Wille ${ }^{2}$ has measured the transport numbers of cupric ions in water and in the presence of excess ammonia. Although in his serjes of solutions the position of equilibrium is not constant, it is apparent that the transport number at inflite dilution of the complex cuprammonium cation is much less than that for the aquo-ion, and, assuming Stokes's law to hold for the large complex cations in aqueous solutions, corresponds to anlionic radius of about 5 A. From data on the relevant bond-lengths, the radius of the cuprammonium cation would be expected to be of the order $3 \cdot 5 \mathrm{~A}$. Hence the complex ion, despite the low charge-density at its surface, is probably able to attract water molecules in aqueous solution; but in strong ammoniacal solutions, there will be less solvation.
It is possible that the peculiar structure of ammoniacal solutions may be of importance in allowing ready diffusion of complex cations. Fajans and Johnson ${ }^{4}$ have discussed the evidence that the ammonium ion fits into the tetrahedral structure of liquid water, and possibly stabilizes it. With other complex-forming molecules such as pyridine or tetramethyl ammonium hydroxide in ammonium salt solutions, the polarographic properties are inferior to those of ammonia - ammonthe polarographic properties are inferior to those of ammonia - ammonis presumably due to the fact that excess of the complex-forming agent must be added to ensure full co-ordination, and the large molecules
mulation must be added to ensure full
distort the solvent structure.

Experimental results suggest that the anion in the supporting electrolyte affects much less the polarographic properties; chloride, electrolyte affects much less the polarographic properties; chioride, buffers for polarographic analysis, and even the larger acetate, perchlor polarographic analysis, and even the larger acetate,

Considerable carbouts Considerable amounts of monovalent and divalent cations may be present in ammoniacal solutions without altering the desirable polarographic features. In a solution molar with respect to ammonia and ion may be repiaced by potassium, without affecting greatly the reduction steps on the curve. However, if the $p H$ of the solution ls to be kept constant, it is necessary to reduce the concentration of ammonia equally with that of the ammonium salt. At $p \mathrm{H}$ values above 10 , divalent metals may be precipitated.

In one respect the supporting electrolytes may even be improved by addition of cations. In polarograms with ammoniacal supportin electrolytes, there is a steep rise of current at about $-1 \cdot 7 \mathrm{~V}$. with respect to the saturated calomel electrode, because the ammonium ion catalyses the deposition of hydrogen and reduces its over-potential. In a molar solution of lithium chloride, the over-potential is about $-1.95 \mathrm{~V}$. If some of the ammonium salt in an ammoniacal buffer is replaced by lithium chloride, an intermediate over-potential value is obtained, and a larger portion of the current-voltage curve is therefore available for analytical purposes. In particular, the diffusion eurrent of the manganese wave, often obscure in ammoniacal buffers, is much clearer in lithium-ammonium mixtures. The diffusion current constants are scarcely affected by this incorporation of lithium into the buffer.

When trace elements in plant material are being estimated polarographically, the cations may be separated from interfering anions by means of synthetic resin adsorbers. The eluted cations may then be estimated in a single polarogram, using an ammoniacal supporting electrolyte. It is not necessary to isolate the trace elements from other cations (calcium, potassium, magnesium) invariably present in plant digests, provided that their total concentration does not exceed that of ammonium ion in the test solution.

This work was done as a member of a team flnanced by the Agricultural Research Council to work on the diagnosis and cure of mineral deficiencles in plants.

East Malling Research Station,

J. P. R. RICHFs.

Botany School,

Cambridge.
Dec. 9.

1 Tyler and Dean, Ind. Eng. Chem. Anal. Ed., 15, 520 (1943).

Lingane, Ind. Eng. Chem. Anal. Ed., 15, Б83 (1943).

Wille, Z. phys. Chem.; A, 171, 93 (1943).

Polarographic Investigations in an Ethylene Glycol Medium

Drspime the many contributions that have been made to the litera. ture of polarography, very few papers have dealt with electrolysis employing a dropping mercury electrode in non-aqueous solvents ${ }^{1}$. Nevertheless, such work is of theoretical interest and may well attain some practical importance in analytical and physical chemistry.

The present studies have been concerned with solutions in carefully purifled, anhydrous ethylene glycol. The solutions have all contained $0.5 \mathrm{gm}$. of potassium chloride in $100 \mathrm{ml}$., and have been protected from atmospheric moisture. It has been found necessary to gas the solutions with dry, oxygen-free hydrogen for two hours, in order to remove the oxygen, which would otherwise give a double wave similar to that obtained in aqueous solutions. Throughout this work, a pool of mercury has been used as the non-polarizable anode, owing to the difficulty of correcting for the liquid junction potential if a standard half-cell were used. The $i R$ drop ${ }^{1}$ in ethylene glycol solution has been
measured and found to be negligible in comparlson with the applied measur

Polarograms of very dilute solutions of a number of anhvdrous salts in the supporting medium have been recorded, and the half-wave potentials $v$. the mercury anode, of cadmium, zinc, thallous, cuprous, cupric and ferrous ions measured. A comparison of these half-wave potentials with those obtained under identical conditions in aqueous solution has shown that the value for a given lon agrees to within \pm 0.02 volt. The current-voltage curves in ethylene glycol have all been well defined, and except in the case of cuprous copper, which exhibited a small maximum, no irregularities have been observed. Quantitative experiments with thallous fon have established that, in accordance with חkovic's equation ${ }^{2}$, the diffusion current is directly proportional to the concentration of the reducible lon, to within the experimental error of \pm 2 per cent. The diffusion current of a given concentration of thallous fon in ethylene glycol solution is smaller than the diffusion current in aqueous solution, in the ratio of 1 to $4 \cdot 3$. A series of experiments have been made on solutions containing a fixed concentration of thallous ion in the presence of the large excess of potassium chloride in solvent mixtures ranging from anhydrous othylene glycol to water. It has been established that the product of the diffusion current and the square root of the viscosity of the
medium is a constant to within an experimental error of \pm 1 per medium is a constant to within an experimental error of
cent, and is independent of the composition of the solvent. 